\title{
Preoperative Patient-Reported Outcomes Measurement Information System Computerized Adaptive Testing (PROMIS CAT) Scores Predict Achievement of Minimum Clinically Important Difference Following Anterior Cruciate Ligament Reconstruction Using an Anchor-Based Methodology
}

\author{
Nikhil R. Yedulla, B.S., Joseph S. Tramer, M.D., Dylan S. Koolmees, B.S., \\ Sreten Franovic, M.S., Kareem G. Elhage, B.S., Vasilios Moutzouros, M.D., and \\ Eric C. Makhni, M.D., M.B.A.
}

\begin{abstract}
Purpose: To determine the change in Patient-Reported Outcomes Measurement Information System Computerized Adaptive Testing (PROMIS CAT) scores for physical function, pain interference, and depression that constitute minimum clinically important difference (MCID) using an anchor-based technique and to identify pre-operative clinical thresholds in anchor-based MCID that predict likelihood of achieving MCID following anterior cruciate ligament (ACL) reconstruction. Methods: Adult patients aged 18 years or older undergoing ACL reconstruction that completed both preoperative and postoperative PROMIS CAT assessments and an anchor-based questionnaire were identified over a 23-month period. Anchor-based MCID was determined for PROMIS CAT forms for physical function (PROMIS PF CAT), pain interference (PROMIS PI CAT), and depression (PROMIS D CAT). Results: A total of 137 patients were included for statistical analysis, with pre-operative PROMIS CAT forms completed $27.9 \pm 31.2$ days before surgery and $492.5 \pm 219.9$ days postoperatively on average. Statistically significant improvements were observed for all PROMIS CAT domains. PROMIS PF CAT improved from $39.5 \pm 8.2$ to $55.0 \pm 9.7(P<.0005)$, PROMIS PI CAT from $59.8 \pm 7.2$ to $48.2 \pm 8.3(P<.0005)$, and PROMIS D CAT from $47.9 \pm 8.8$ to $41.5 \pm 8.6(P<.0005)$. Anchor-based MCID for each PROMIS CAT form was calculated to be $+4.5,-5.4$, and -4.1 for PROMIS PF CAT, PROMIS PI CAT, and PROMIS D CAT, respectively. Mean difference between preoperative and postoperative PROMIS CAT scores exceeded MCID for all domains. The percentage of patients achieving MCID for PROMIS PF CAT, PROMIS PI CAT, and PROMIS D CAT was $85 \%, 72 \%$, and 55\%, respectively. After introduction of $95 \%$ specificity cutoffs, the percentage of patients achieving MCID for PROMIS PF CAT, PROMIS PI CAT, and PROMIS D CAT increased to $100 \%$ ( $<35.6$ cutoff score), $92 \%$ ( $>65.7$ cutoff score), and $83 \%$ ( $>57.5$ cutoff score), respectively. Conclusions: According to anchor-based analysis of PROMIS CAT MCID, ACL reconstruction is effective in improving physical function, pain interference, and depression symptoms. In addition, preoperative PROMIS CAT scores can predict the likelihood of achieving MCID postoperatively. Level of Evidence: Level IV, prognostic case series.
\end{abstract}

$\mathbf{P}$ atient-reported outcome measures (PROMs) are used in orthopaedic surgery to assess clinical outcomes from the patient's perspective. The National

From the Department of Orthopedic Surgery, Henry Ford Health System, Detroit, Michigan, U.S.A.

The authors report that they have no conflicts of interest in the authorship and publication of this article. Full ICMJE author disclosure forms are available for this article online, as supplementary material.

Received April 2, 2021; accepted September 1, 2021.

Address correspondence to Eric C. Makhni, M.D., M.B.A., Department of Orthopaedic Surgery, Henry Ford Health System, 2799 W. Grand Blvd., Detroit, MI 48202.E-mail: ericmakhnimd@gmail.com
Institutes of Health Patient-Reported Outcomes Measurement System (PROMIS) is a standardized PROM that has emerged as an increasingly popular tool for

(C) 2021 Published by Elsevier on behalf of the Arthroscopy Association of North America. This is an open access article under the CC BY-NC-ND license (http://creativecommons.org/licenses/by-nc-nd/4.0/).

2666-061X/21470

https://doi.org/10.1016/j.asmr.2021.09.004 
measuring patient reported outcomes in response to treatment. ${ }^{1}$ In particular, the computer adaptive test (CAT) version of these forms has demonstrated favorable survey and psychometric properties when compared with "legacy" PROMs, such as the Health Assessment Questionnaire or Medical Outcome Study Short Form-36 Survey, in orthopaedic sports medicine patient populations due to efficient data collection involving fewer questions..$^{2-5}$ These CAT questionnaires use item response theory to customize question delivery based on real-time patient answers, leading to 4to 12-item forms that correlate strongly with other PROMs, while remaining highly reliable. ${ }^{3,4}$ In addition, PROMIS CAT health domains allow for a multifaceted evaluation of patient outcomes by interpreting pain interference (PROMIS PI CAT), physical function (PROMIS PF CAT), and depression (PROMIS D CAT) separately. ${ }^{1,6,7}$

Recently, PROMIS CAT measures have demonstrated utility in measuring responsiveness to treatment following various orthopedic interventions. ${ }^{8-10}$ However, postoperative statistical score improvements may not indicate clinical improvement. To understand whether a patient has improved clinically (as opposed to statistically), the score change correlating to the minimal clinically important difference (MCID) must be identified. ${ }^{11}$ There are 2 techniques commonly used to identify the score change needed for achieving MCID. The first technique, called the distribution-based (DB) technique, defines the MCID as one half of the standard deviation of the preoperative scores from a cohort. ${ }^{12}$ The second technique, called the anchor-based $(\mathrm{AB})$ technique, correlates score changes with anchor questions that denote clinical improvement from the patient's perspective. ${ }^{13,14}$ The $\mathrm{AB}$ technique is considered more precise, as score changes are correlated to actual subjective metrics of symptom improvement, as opposed to statistical correlations in the DB technique. ${ }^{11,13,14}$ Despite this recognition, the $\mathrm{AB}$ technique is less frequently reported on when compared with the DB technique due to the increased challenge of collecting postoperative anchor assessments. ${ }^{15}$

In patients undergoing anterior cruciate ligament reconstruction (ACLR), the MCID for PROMIS CAT measures have been identified previously using a DB technique. Chen et al. ${ }^{16}$ demonstrated that PROMIS PF CAT, PROMIS PI CAT, and PROMIS D CAT scores improved significantly in patients who underwent ACLR, and that preoperative scores were highly predictive of postoperative outcomes. ${ }^{16}$ In addition, preoperative score ranges for PROMIS CAT assessments have been correlated to likelihood of achieving MCID. ${ }^{17-19}$ This knowledge can be used in preoperative shared decision-making with patients. ${ }^{20}$ However, the MCID for PROMIS CAT measures using an $\mathrm{AB}$ technique has not been studied for patients undergoing ACLR, and neither have the score ranges that correlate with likelihood of achieving MCID. The AB method improves upon the $\mathrm{DB}$ method by associating the pre- and postoperative difference in outcome measure score to a meaningful external anchor question, which represents the patient's perspective, thereby optimally representing clinical changes from a patient's perspective. ${ }^{15,16,21,22}$

The purposes of this study were to determine the change in PROMIS CAT scores (for PF, PI, and D) that constitute MCID using an $\mathrm{AB}$ technique and to identify preoperative clinical thresholds in $\mathrm{AB}$ MCID that predict likelihood of achieving MCID following ACL reconstruction. Given that the defined standard deviation of PROMIS CAT forms are defined to be 10 points, we hypothesized that AB MCIDs would similarly approximate 5 points. ${ }^{23}$ Moreover, we hypothesized that patients would be more likely to achieve MCID for physical function if they exhibit low preoperative PROMIS PF CAT scores, whereas those with high preoperative PROMIS PI CAT and PROMIS D CAT scores would be more likely to achieve MCID for those respective domains.

\section{Methods}

This investigation was a retrospective case series study that received institutional review board approval from Henry Ford Hospital. Patients undergoing primary ACLR by 1 of 2 fellowship-trained sports surgeons were identified over a 23-month period from July 2017 to June 2019. Three PROMIS CAT forms (PROMIS PF CAT, PROMIS PI CAT, PROMIS D CAT) were prospectively collected from all patients. PROMIS CAT forms were administered on an iPad (Apple Inc., Cupertino, CA) or via e-mail message by using REDCap, an electronic, secure platform for recording PROMIS CAT forms (REDCap, Vanderbilt University, Nashville, TN). Inclusion criteria were adult patients aged 18 years or older who completed all preoperative PROMIS forms as well as postoperative PROMIS forms and anchor questionnaire items. Patients were excluded if they were unable to answer PROMs (e.g., language barrier), did not complete preoperative PROMIS forms, or did not have an available e-mail address for postoperative PROMIS form and anchor questionnaire administration. In addition, a chart review was conducted to record demographic factors such as age, ethnicity, sex, and smoking status, as well as surgical factors such as side of surgery, graft type, meniscal injury, and concomitant procedures.

PROMIS CAT forms were collected preoperatively as well as postoperatively. Three clinical anchor questions inquiring about patient perception of improvement in physical function, pain, and mental health relative to presurgery were administered electronically with each postoperative PROMIS CAT survey. The anchor questionnaire used was adapted from previously validated 
Anchor Question 1: How has the functioning of your knee improved since your surgical procedure?

Gotten worse

About the same

A bit better

Significantly better

Complete improvement
No Significant Improvement Group

Significant Improvement Group
Fig 1. Overview of anchor questions used for minimal clinically important difference (MCID) patient groupings. For calculation of MCID, differences between the "no significant improvement group" and the "significant improvement group" were assessed.

Anchor Question 2: Compared to before surgery, how much does pain (in your affected joint) impact your life today?

More than before

About the same

A bit less than before

Significantly less than before

No impact anymore
No Significant Improvement Group

Significant Improvement Group

Anchor Question 3: How has your mental health changed since your surgical procedure?

Gotten worse

About the same

A bit better

Significantly better

Complete improvement
No Significant Improvement Group

Significant Improvement Group anchor questions in the literature and included question items that respectively assessed physical function, pain, and depression as displayed in Fig 1, with 2 separate groups representing "significant improvement" and "no significant improvement" determined for each question item based on responses. ${ }^{24,25}$ According to PROMIS CAT scoring, a score of 50 represents the mean score of the reference population within any given health domain, with 10 points denoting one standard deviation. All PROMIS CAT forms were administered by research assistants in the institution's Department of Orthopedics who were either current medical students or full-time employees currently taking a gap year from medical school to conduct research.
Preoperative PROMIS forms considered were administered at the patient's clinic visit closest to the date of their surgery, whereas postoperative PROMIS forms and anchor questionnaire items were collected either at clinic visits or remotely via e-mail with the most recent forms being considered.

\section{Statistical Analysis}

Paired samples $t$ tests were used to compare preoperative and postoperative PROMIS CAT scores. MCID was calculated according to AB methodology. AB MCID was determined using independent samples $t$ tests on anchor question responses to determine statistical differences between responses indicating "no significant 


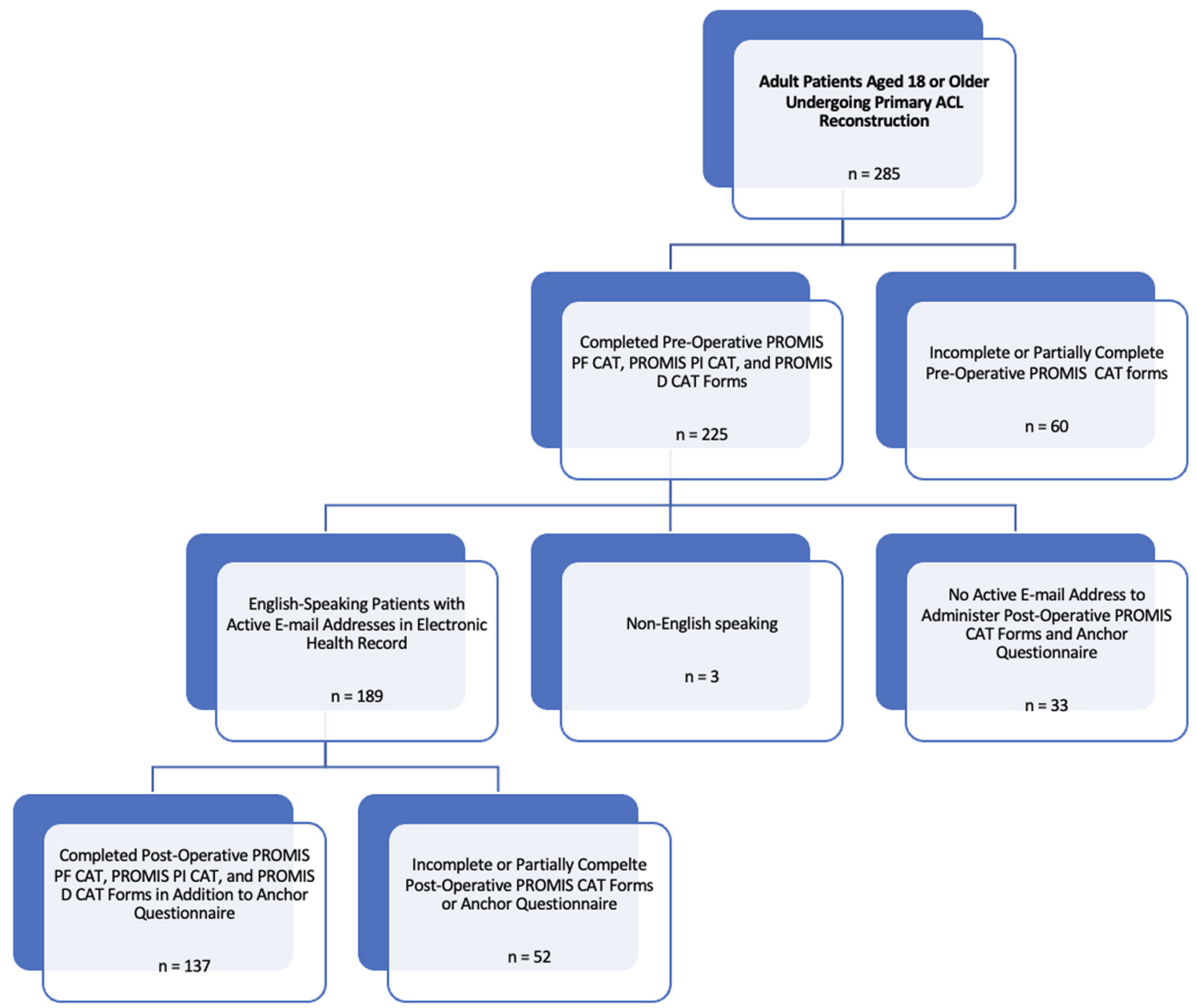

Fig 2. Enrolled patient cohort with inclusion and exclusion criteria. (ACL, anterior cruciate ligament; PROMIS D CAT, Patient-Reported Outcomes Measurement Information System Computer Adaptive Test forms for Depression; PROMIS PF CAT, Patient-Reported Outcomes Measurement Information System Computer Adaptive Test form for Physical Function; PROMIS PI CAT, Patient-Reported Outcomes Measurement Information System Computer Adaptive Test forms for Pain Interference.

improvement" and "significant improvement." Once confirmed, respective mean PROMIS CAT domain changes were identified as the MCID. In addition, all PROMIS CAT scores were assessed for discriminatory ability in predicting a postoperative improvement (of the same domain) that reached the threshold of MCID through use of receiver operating characteristic curve analyses, with overall accuracy summarized using the area under the ROC curve (area under the curve [AUC]). AUC values were interpreted as follows: weak (0.5-0.599), moderate (0.6-0.699), strong (0.7-0.799), and excellent $(>0.8) .{ }^{13}$ Prognostic cutoffs for achievement, or failure to achieve clinically significant outcomes, were set using $95 \%$ specificity. All analyses used a significance level of $5 \%$. SPSS software was used for all statistical analyses (IBM Corp. Released 2017. IBM SPSS Statistics for Windows, Version 25.0. IBM Corp., Armonk, NY).

\section{Results}

One hundred eighty-nine patients who underwent ACLR and fully completed preoperative PROMIS CAT forms who were available at follow-up were assessed for inclusion. A total of 137 patients completed all preoperative PROMIS forms as well as postoperative PROMIS forms and anchor questionnaire items and were included for statistical analysis. Fig 2 displays the included and excluded patients undergoing primary ACLR during the study period. The patient population consisted of 82 male subjects $(60 \%)$ and 55 female subjects $(40 \%)$, with an average age of $30.3 \pm 11.4$ years. Preoperative PROMIS CAT forms were completed $27.9 \pm 31.2$ days before surgery and $492.5 \pm$ 219.9 days post-operatively on average. Complete demographic characteristics are summarized in Table 1.

Across the entire cohort, statistically significant improvements were observed for all analyzed PROMIS 
Table 1. ACLR Cohort Demographics

\begin{tabular}{lrc}
\hline Average age, y & \multicolumn{2}{c}{$30.3 \pm 11.4$} \\
Sex & Total & $\%$ \\
Male & 82 & 60 \\
Ethnicity & Total & $\%$ \\
Not Hispanic/Latino & 120 & 87.6 \\
Hispanic/Latino & 8 & 5.8 \\
Declined to answer/unknown & 9 & 6.6 \\
Smoker & Total & $\%$ \\
Never & 115 & 83.9 \\
Current & 12 & 8.8 \\
Former & 10 & 7.3 \\
Side of surgery & Total & $\%$ \\
Bone-tendon-patellar-bone & 102 & 74.5 \\
Hamstring & 31 & 22.6 \\
Quadriceps & 3 & 2.2 \\
Unspecified & 1 & 0.7 \\
Graft Source & Total & $\%$ \\
Allograft & 9 & 6.6 \\
Concomitant procedure & Total & $\%$ \\
Medial meniscectomy & 17 & 12.4 \\
Lateral meniscal repair & 9 & 6.6 \\
Medial meniscal repair & 16 & 11.7 \\
Meniscal debridement & 1 & 0.7 \\
Medial cruciate ligament repair & 3 & 2.2 \\
Posterior cruciate ligament repair & 1 & 0.7 \\
Lateral cruciate ligament repair & 2 & 1.5 \\
Allograft augment & 1 & 0.7 \\
\hline ACLR anterior cruciate ligament reconstruction &
\end{tabular}

ACLR, anterior cruciate ligament reconstruction.

CAT domains. PROMIS PF CAT improved from $39.5 \pm$ 8.2 to $55.0 \pm 9.7(P<.0005)$, PROMIS PI CAT improved from $59.8 \pm 7.2$ to $48.2 \pm 8.3(P<.0005)$, and PROMIS D CAT improved from $47.9 \pm 8.8$ to 41.5 $\pm 8.6(P<.0005)$. With AB methodology, the MCID for each PROMIS CAT form was calculated to be +4.5, -5.4, and -4.1, for PROMIS PF CAT, PROMIS PI CAT, and PROMIS D CAT, respectively. Average change in PROMIS CAT scores before and after surgery exceeded MCID for all domains (Table 2). AUC values were 0.756 (PROMIS PF CAT), 0.728 (PROMIS PI CAT), and 0.761 (PROMIS D CAT), indicating strong predictive ability.

Overall, the percent of patients who met MCID for PROMIS PF CAT, PROMIS PI CAT, and PROMIS D CAT was $85 \%, 72 \%$, and $55 \%$, respectively. Post hoc analysis was performed in order to determine prognostic cutoff thresholds for achieving or failing to achieve MCID using $95 \%$ specificity. Application of prognostic cutoffs to presurgical PROMIS CAT scores improved the accuracy of predicting patients that would achieve or fail to achieve MCID, with results summarized in Table 3. For example, in PROMIS PI CAT, a pre-operative score $>65.7$ resulted in $92 \%$ of patients achieving MCID, whereas a score of $<51.7$ resulted in $67 \%$ of patients failing to achieve MCID. In addition, for PROMIS PF CAT, a preoperative score of $<35.6$ resulted in $100 \%$ of patients achieving MCID, while a score of $>50.7$ resulted in $40 \%$ of patients failing to achieve MCID.

\section{Discussion}

ACLR resulted in significant improvements in PROMIS PF CAT, PROMIS PI CAT, and PROMIS D CAT scores. The majority of patients exceeded AB MCID in PROMIS PF CAT, PROMIS PI CAT, and PROMIS D CAT following surgery. Using prognostic cutoffs, preoperative scores were able to accurately predict the postoperative response to surgery.

PROMs are essential tools for evaluating patient response to intervention and providing objective measurements of the patient experience. PROMIS has been validated as a reliable patient reported outcome metric compared to other PROMs used in knee surgery, and its increasing use is largely attributed to not only this favorable correlation but also decreased survey burden for patients. ${ }^{26-30}$ Furthermore, PROMIS PF CAT, PROMIS PI CAT, and PROMIS D CAT have all been validated in assessing outcomes in ACLR patient populations in particular. ${ }^{31-33}$ The present investigation was able to define the magnitude of MCID for three relevant PROMIS CAT domains in patients undergoing ACLR by using an $A B$ method. In addition, average PROMIS CAT scores had statistically significant improvement following ACLR with improvements exceeding $A B$ MCID. Similar findings were demonstrated in a previous study by Chen et al., ${ }^{16}$ which assessed PROMIS CAT MCID in ACLR patients according to the DB methodology. The authors also found that the average change in PROMIS CAT scores exceeded the MCID following their procedures. ${ }^{16}$ While the investigation by Chen et al. provides essential information regarding patient response to ACLR, a weakness of the DB method is the focus on statistical significance and consequential failure to incorporate the patient's perspective of clinically important change. ${ }^{14,34}$ The $\mathrm{AB}$ method addresses the shortcomings of the DB method by linking the pre- and postoperative difference in outcome measure score to a meaningful external anchor question, which represents the patient's perspective. ${ }^{21,22}$ Although DB MCID is a valuable method for predicting clinically meaningful improvement, $\mathrm{AB}$ MCID is shown to better represent patient-centric clinical changes. ${ }^{15,35}$

While many patients experience positive surgical outcomes following ACLR, there remains a subset of patients who do not return to preinjury levels of physical function. Previous investigations by Ardern et al. $^{36}$ and Rodríguez-Roize et al. ${ }^{37}$ indicate that only $52 \%$ and $65 \%$ of athletes undergoing ACLR return to their preinjury performance levels, respectively. Thus, it is important to identify patients who are at risk for poor outcomes to optimize treatment. One such strategy for optimizing outcomes is use of preoperative PROMs to gauge patients' degree of physical and mental impairment. The present investigation revealed that applying 
Table 2. PROMIS CAT Scores and AB MCID

\begin{tabular}{|c|c|c|c|c|c|}
\hline \multirow{2}{*}{$\begin{array}{c}\text { PROMIS } \\
\text { CAT Domain }\end{array}$} & \multicolumn{3}{|c|}{ Average Scores } & \multicolumn{2}{|c|}{ AB MCID } \\
\hline & Preoperative & Postoperative & Change & MCID & ROC \\
\hline PROMIS PF CAT & $39.5 \pm 8.2$ & $55 \pm 9.7$ & +15.5 & +4.5 & .756 \\
\hline PROMIS PI CAT & $59.8 \pm 7.2$ & $48.2 \pm 8.3$ & -11.6 & -5.4 & .728 \\
\hline PROMIS D CAT & $47.9 \pm 8.8$ & $41.5 \pm 8.6$ & -6.4 & -4.1 & .761 \\
\hline
\end{tabular}

AB MCID, minimum clinically important difference calculated according to anchor-based methodology; PROMIS D CAT, PatientReported Outcomes Measurement Information System Computer Adaptive Test forms for Depression; PROMIS PF CAT, PatientReported Outcomes Measurement Information System Computer Adaptive Test form for Physical Function; PROMIS PI CAT, PatientReported Outcomes Measurement Information System Computer Adaptive Test forms for Pain Interference; ROC, receiver operating characteristic.

preoperative score cutoffs in all three PROMIS CAT domains enhanced the ability to predict patients who would achieve or fail to achieve MCID following surgery. This has also been evaluated in orthopaedic foot and ankle patients, as Ho et al. ${ }^{18}$ used similar methodology to provide clinical cutoffs that aid in preoperative decision making in a variety of foot and ankle conditions. The aforementioned study by Chen et al. also found that preoperative clinical cutoffs were beneficial in predicting post-operative outcomes following ACLR using the DB MCID. ${ }^{6}$ As in our study, both of these investigations also noted that worse baseline physical function and pain scores portended to a higher likelihood of achieving MCID following surgery. Still, it is important to recognize how these patients with lower baseline function did not necessarily achieve similar post-operative levels of function to their counterparts with higher initial function, even though they were more likely to achieve MCID.

The practical application of PROMs to clinical decision making remains a challenge. PROMIS CAT has been demonstrated to have strong responsiveness and minimal floor and ceiling effects in patients with knee pathology. ${ }^{7,38}$ Thus, the use of PROMIS CAT forms as well as the findings of the present investigation can be beneficial in the shared decision-making process with patients who have suffered from ACL tears. For example, a moderately active 50-year-old female with an ACL tear who has pre-operative PROMIS CAT scores consistent with high physical function (PROMIS PF CAT $>50.7$ ) and low pain interference (PROMIS PI CAT $<51.7)$ based on the calculated prognostic cutoffs may not receive a significant clinical benefit following ACLR and resultantly can be educated on alternative treatment options to surgical intervention. ${ }^{16}$ Conversely, a patient with preoperative scores that fall below the cutoff for PROMIS PF CAT $(<35.6)$ and above in PROMIS PI CAT $(>65.7)$ may have a very high preoperative likelihood of exceeding MCID after surgery. While PROMs should not be the primary driver in surgical decision making, they offer an important added measure when making shared decisions with our patients while considering other important variables such as age and activity level. Moreover, pre-operative PROM assessment in this capacity allows surgeons an opportunity to evaluate surgical appropriateness based on the individual patient.

\section{Limitations}

There were limitations to this investigation. All enrolled subjects were English-speaking patients at a metropolitan academic center, thereby potentially limiting the external validity of findings given the lack of representation across different healthcare modalities. However, the general diversity of the enrolled patient population in terms of age, sex, and ethnicity mitigates this limitation to a significant degree. In addition, only patients who completed all PROMIS CAT and anchor questionnaire forms were included in final analysis, thus limiting the total population based on follow-up and willingness to complete forms at each visit. Incomplete patient participation potentially leads to an enrolled cohort that may not be fully representative of the general clinic population. Furthermore, there were differences in ACLR surgical technique and postoperative rehabilitation within the enrolled, both of which are variable factors that have been shown to potentially influence postoperative outcomes that our study did not account for. Regardless, previous literature supports how significant variation exists in postoperative rehabilitation protocols, supporting how our findings are reflective of real-life patient populations. ${ }^{39}$

Table 3. Prognostic Cutoffs for PROMIS CAT Domains

\begin{tabular}{lcccc}
\hline & \multicolumn{2}{c}{ Achievement of AB MCID } & \multicolumn{2}{c}{ Failure to Achieve AB MCID } \\
\cline { 2 - 4 } PROMIS CAT Domain & Pre-Cutoff & Post-Cutoff & & Pre-Cutoff \\
\hline PROMIS PF CAT & $85 \%$ & $100 \%(<35.6)$ & $15 \%$ & \\
PROMIS PI CAT & $72 \%$ & $92 \%(>65.7)$ & $28 \%$ & $67 \%(<51.7)$ \\
PROMIS D CAT & $55 \%$ & $83 \%(>57.5)$ & $45 \%$ & $83 \%(<40.5)$ \\
\hline
\end{tabular}

AB MCID, minimum clinically important difference calculated according to anchor-based methodology; PROMIS D CAT, Patient-Reported Outcomes Measurement Information System Computer Adaptive Test forms for Depression; PROMIS PF CAT, Patient-Reported Outcomes Measurement Information System Computer Adaptive Test form for Physical Function; PROMIS PI CAT, Patient-Reported Outcomes Measurement Information System Computer Adaptive Test forms for Pain Interference. 
Lastly, there are some inherent limitations to the clinical interpretation of MCID as calculated in this study. For certain orthopaedic procedures such as hip arthroscopy, there are low levels of patient satisfaction between 25 and $30 \%$ as reflected by the patient acceptable symptom state (PASS), despite at least $90 \%$ of patients achieving MCID in various outcome measures and thus achieving improvement in health status. ${ }^{40}$ Such findings indicate how achieving MCID may not represent true satisfaction with clinical improvement, which may also be a discrepancy that applies to our study given that we do not assess PASS to show otherwise. ${ }^{41}$ Regardless, we still feel that our study is valuable given how MCID is a reliable indicator of clinical improvement in its own right, and how future follow-up studies can focus on evaluating PASS.

\section{Conclusions}

According to AB analysis of PROMIS CAT MCID, ACL reconstruction is effective in improving physical function, pain interference, and depression symptoms. In addition, preoperative PROMIS CAT scores can predict the likelihood of achieving MCID postoperatively.

\section{References}

1. Cella D, Yount S, Rothrock N, et al. The Patient-Reported Outcomes Measurement Information System (PROMIS): Progress of an NIH Roadmap cooperative group during its first two years. Med Care 2007;45:S3-S1 1 (5 Suppl 1).

2. Patterson BM, Orvets ND, Aleem AW, et al. Correlation of Patient-Reported Outcomes Measurement Information System (PROMIS) scores with legacy patient-reported outcome scores in patients undergoing rotator cuff repair. J Shoulder Elbow Surg 2018;27:S17-S23.

3. Fries JF, Bruce B, Cella D. The promise of PROMIS: Using item response theory to improve assessment of patientreported outcomes. Clin Exp Rheumatol 2005;23:S53-S57 (5 suppl 39).

4. Fries JF, Witter J, Rose M, Cella D, Khanna D, MorganDeWitt E. Item response theory, computerized adaptive testing, and PROMIS: Assessment of physical function. J Rheumatol 2014;41:153-158.

5. Gulledge CM, Smith DG, Ziedas A, Muh SJ, Moutzouros V, Makhni EC. Floor and ceiling effects, time to completion, and question burden of PROMIS CAT domains among shoulder and knee patients undergoing nonoperative and operative treatment. JB JS Open Access 2019;4:e0015.1-7.

6. Beleckas CM, Guattery J, Chamberlain AM, Khan T, Kelly MP, Calfee RP. Using PROMIS measures to understand the relationship between improvement in physical function and depressive symptoms. J Am Acad Orthop Surg 2018;26:e511-e518.

7. Lu Y, Beletsky A, Nwachukwu BU, et al. Performance of PROMIS Physical Function, Pain Interference, and Depression Computer Adaptive Tests instruments in patients undergoing meniscal surgery. Arthrosc Sports Med Rehabil 2020;2:e451-e459.
8. Fisk F, Franovic S, Tramer JS, et al. PROMIS CAT forms demonstrate responsiveness in patients following arthroscopic rotator cuff repair across numerous health domains. J Shoulder Elbow Surg 2019;28:2427-2432.

9. Bernstein DN, Houck JR, Mahmood B, Hammert WC. Responsiveness of the PROMIS and its concurrent validity with other region- and condition-specific PROMs in patients undergoing carpal tunnel release. Clin Orthop Relat Res 2019;477:2544-2551.

10. Hung M, Saltzman CL, Greene T, et al. The responsiveness of the PROMIS instruments and the qDASH in an upper extremity population. I Patient Rep Outcomes 2017;1:12.

11. Jaeschke R, Singer J, Guyatt GH. Measurement of health status. Ascertaining the minimal clinically important difference. Control Clin Trials 1989;10:407-415.

12. McGlothlin AE, Lewis RJ. Minimal clinically important difference: Defining what really matters to patients. JAMA 2014;312:1342-1343.

13. Lydick E, Epstein RS. Interpretation of quality of life changes. Qual Life Res 1993;2:221-226.

14. Crosby RD, Kolotkin RL, Williams GR. Defining clinically meaningful change in health-related quality of life. J Clin Epidemiol 2003;56:395-407.

15. Revicki D, Hays RD, Cella D, Sloan J. Recommended methods for determining responsiveness and minimally important differences for patient-reported outcomes. J Clin Epidemiol 2008;61:102-109.

16. Chen RE, Papuga MO, Voloshin I, et al. Preoperative PROMIS scores predict postoperative outcomes after primary ACL reconstruction. Orthop J Sports Med 2018;6: 2325967118771286.

17. Chen RE, Papuga MO, Nicandri GT, Miller RJ, Voloshin I. Preoperative Patient-Reported Outcomes Measurement Information System (PROMIS) scores predict postoperative outcome in total shoulder arthroplasty patients. J Shoulder Elbow Surg 2019;28:547-554.

18. Ho B, Houck JR, Flemister AS, et al. Preoperative PROMIS scores predict postoperative success in foot and ankle patients. Foot Ankle Int 2016;37:911-918.

19. Franovic S, Kuhlmann NA, Pietroski A, et al. Preoperative patient-centric predictors of postoperative outcomes in patients undergoing arthroscopic meniscectomy. Arthroscopy 2021;37:964-971.

20. Wilson I, Bohm E, Lübbeke A, et al. Orthopaedic registries with patient-reported outcome measures. EFORT Open Rev 2019;4:357-367.

21. Rai SK, Yazdany J, Fortin PR, Aviña-Zubieta JA. Approaches for estimating minimal clinically important differences in systemic lupus erythematosus. Arthritis Res Ther 2015;17.

22. Wright A, Hannon J, Hegedus EJ, Kavchak AE. Clinimetrics corner: A closer look at the minimal clinically important difference (MCID). J Man Manip Ther 2012;20:160-166.

23. Cella D, Choi S, Garcia S, et al. Setting standards for severity of common symptoms in oncology using the PROMIS item banks and expert judgment. Qual Life Res 2014;23:2651-2661.

24. Cvetanovich GL, Gowd AK, Liu JN, et al. Establishing clinically significant outcome after arthroscopic rotator cuff repair. J Shoulder Elbow Surg 2019;28:939-948. 
25. Nwachukwu BU, Beck EC, Kunze KN, Chahla J, Rasio J, Nho SJ. Defining the clinically meaningful outcomes for arthroscopic treatment of femoroacetabular impingement syndrome at minimum 5-year follow-up. Am J Sports Med 2020;48:901-907.

26. Hajewski CJ, Baron JE, Glass NA, et al. Performance of the patient-reported outcome measurement information system in patients with patellofemoral instability. Orthop $J$ Sports Med 2020;8:2325967120915540.

27. Hancock KJ, Glass N, Anthony CA, et al. Performance of PROMIS for healthy patients undergoing meniscal surgery. J Bone Joint Surg Am 2017;99:954-958.

28. Baumhauer JF. Patient-reported outcomes-are they living up to their potential? N Engl J Med 2017;377:6-9.

29. Kadri O, Jildeh TR, Meldau JE, et al. How long does it take for patients to complete PROMIS scores? An assessment of PROMIS CAT questionnaires administered at an ambulatory sports medicine clinic. Orthop J Sports Med 2018;6: 2325967118791180.

30. Senders A, Hanes D, Bourdette D, Whitham R, Shinto L. Reducing survey burden: Feasibility and validity of PROMIS measures in multiple sclerosis. Mult Scler 2014;20:1 102-1111.

31. Papuga MO, Beck CA, Kates SL, Schwarz EM, Maloney MD. Validation of GAITRite and PROMIS as highthroughput physical function outcome measures following ACL reconstruction. J Orthop Res 2014;32:793-801.

32. Scott EJ, Westermann R, Glass NA, Hettrich C, Wolf BR, Bollier MJ. Performance of the PROMIS in patients after anterior cruciate ligament reconstruction. Orthop J Sports Med 2018;6:2325967118774509.

33. Gulledge CM, Koolmees D, Smith DG, et al. The PROMIS CAT Demonstrates responsiveness in patients after ACL reconstruction across numerous health domains. Orthop $J$ Sports Med 2021;9:2325967120979991.

34. Copay AG, Subach BR, Glassman SD, Polly DW, Schuler TC. Understanding the minimum clinically important difference: A review of concepts and methods. Spine J 2007;7:541-546.

35. Chan HY, Chen JY, Zainul-Abidin S, Ying H, Koo K, Rikhraj IS. Minimal clinically important differences for American Orthopaedic Foot \& Ankle Society Score in hallux valgus surgery. Foot Ankle Int 2017;38:551-557.

36. Ardern CL, Taylor NF, Feller JA, Webster KE. Fifty-five per cent return to competitive sport following anterior cruciate ligament reconstruction surgery: An updated systematic review and meta-analysis including aspects of physical functioning and contextual factors. Br J Sports Med 2014;48:1543-1552.

37. Rodríguez-Roiz JM, Caballero M, Ares O, Sastre S, Lozano L, Popescu D. Return to recreational sports activity after anterior cruciate ligament reconstruction: A one- to six-year follow-up study. Arch Orthop Trauma Surg 2015;135:1117-1122.

38. Lee AC, Driban JB, Price LL, Harvey WF, Rodday AM, Wang C. Responsiveness and minimally important differences for 4 Patient-Reported Outcomes Measurement Information System Short Forms: Physical Function, Pain Interference, Depression, and Anxiety in knee osteoarthritis. J Pain 2017;18:1096-1110.

39. Makhni EC, Crump EK, Steinhaus ME, et al. Quality and variability of online available physical therapy protocols from academic orthopaedic surgery programs for anterior cruciate ligament reconstruction. Arthroscopy 2016;32: 1612-1621.

40. Levy DM, Kuhns BD, Chahal J, Philippon MJ, Kelly BT, Nho SJ. Hip arthroscopy outcomes with respect to patient acceptable symptomatic state and minimal clinically important difference. Arthroscopy 2016;32:1877-1886.

41. Harris JD, Brand JC, Cote MP, Faucett SC, Dhawan A. Research Pearls: The significance of statistics and perils of pooling. part 1: Clinical versus statistical significance. Arthroscopy 2017;33:1102-1112. 